HUPD-92-10

UB-ECM-PF 92/29

October 1992

\title{
Gravitational phase transitions in infrared quantum gravity
}

\author{
E. ElizALDE円 \\ Department E.C.M., Faculty of Physics, University of Barcelona, \\ Diagonal 647, 08028 Barcelona, Spain \\ and \\ S.D. Odintsova \\ Department of Physics, Faculty of Science, Hiroshima University, \\ Higashi-Hiroshima 724, Japan
}

\begin{abstract}
The conformal anomaly induced sector of four-dimensional quantum gravity (infrared quantum gravity) — which has been introduced by Antoniadis and Mottola - is here studied on a curved fiducial background. The one-loop effective potential for the effective conformal factor theory is calculated with accuracy, including terms linear in the curvature. It is proven that a curvature induced phase transition can actually take place. An estimation of the critical curvature for different choices of the parameters of the theory is given.
\end{abstract}

\footnotetext{
${ }^{1}$ E-mail address: eli @ ebubecm1.bitnet

${ }^{2}$ On sabbatical leave from Tomsk Pedagogical Institute, 634041 Tomsk, Russia. E-mail address: odintsov @ theo.phys.sci.hiroshima-u.ac.jp
} 
In spite of the considerable efforts invested, there has been yet no definite success in the construction of a consistent theory of four-dimensional (4d) quantum gravity (for a review, see [1]). A very interesting approach for the description of $4 \mathrm{~d}$ quantum gravity at large distances (the so-called infrared quantum gravity) has been developed recently by Antoniadis and Mottola [2]. Their line of reasoning has been borrowed from that followed in two-dimensional gravity, where the anomaly induced dynamics are already completly different from the ones corresponding to the classical theory. In principle, the results of [2] provide a very natural framework for a dynamical solution of the cosmological constant problem [3].

Let us briefly review the basic details of the effective conformal factor dynamics (namely, the conformal sector of $4 \mathrm{~d}$ gravity) [2]. It is well-known that the general form of the conformal anomaly for free conformally invariant fields is

$$
T_{\mu}^{\mu}=b\left(C_{\mu \nu \alpha \beta}^{2}+\frac{2}{3} \square R\right)+b^{\prime} G+b^{\prime \prime} \square R, \quad d=4,
$$

where $C_{\mu \nu \alpha \beta}$ is the Weyl tensor, $R$ the curvature scalar and $G$ the Gauss-Bonnet invariant. Now, as in two dimensions [4], the conformal anomaly induces a new action. Choosing the conformal parametrization

$$
g_{\mu \nu}(x)=e^{2 \sigma(x)} \bar{g}_{\mu \nu}(x),
$$

where $\bar{g}_{\mu \nu}(x)$ is a fixed fiducial metric, one can integrate over the conformal anomaly and get the following action —which has been proposed as the starting point for $4 \mathrm{~d}$ quantum gravity $[2,5]-$

$$
\begin{aligned}
S_{\text {anom }} & =\int d^{4} x \sqrt{-\bar{g}}\left\{b \sigma \bar{C}_{\mu \nu \alpha \beta}^{2}+2 b^{\prime} \sigma\left[\square^{2}+2 \bar{R}^{\mu \nu} \bar{\nabla}_{\mu} \bar{\nabla}_{\nu}-\frac{2}{3} \bar{R} \square+\frac{1}{3}\left(\bar{\nabla}^{\mu} \bar{R}\right) \bar{\nabla}_{\mu}\right] \sigma\right. \\
& \left.+b^{\prime}\left(\bar{G}-\frac{2}{3} \square \bar{R}\right) \sigma-\frac{1}{12}\left[b^{\prime \prime}+\frac{2}{3}\left(b+b^{\prime}\right)\right]\left[\bar{R}-6 \square \sigma-6\left(\bar{\nabla}_{\mu} \sigma\right)\left(\bar{\nabla}^{\mu} \sigma\right)\right]^{2}\right\} .
\end{aligned}
$$

Here $b^{\prime \prime}$ is arbitrary, since it can be changed by adding a local $R^{2}$-term to the action, and

$$
\begin{aligned}
b & =\frac{1}{120(4 \pi)^{2}}\left(N_{S}+6 N_{F}+12 N_{V}-8\right)+\frac{199}{30(4 \pi)^{2}} \\
b^{\prime} & =-\frac{1}{360(4 \pi)^{2}}\left(N_{S}+11 N_{F}+62 N_{V}-28\right)-\frac{87}{20(4 \pi)^{2}} .
\end{aligned}
$$

The contribution of the scalar $(S)$, vector $(V)$ and fermion $(F)$ to the conformal anomaly has been known long ago (see, for example, [8]). The last term in brackets in (4) gives the contribution of the $\sigma$ field to the conformal anomaly [6]. The last term in both $b$ and $b^{\prime}$ is the gravitation/ghost contribution coming from the Weyl gravity action [7].

Now — as it is done in 2d gravity - one should add to the action (3) the classical EinsteinHilbert action in the parametrization (2), thus obtaining

$$
S_{c l}=\frac{1}{2 k} \int d^{4} x \sqrt{-\bar{g}} e^{2 \sigma}\left(\bar{R}-6 \square \sigma-6 \bar{\nabla}^{\mu} \sigma \bar{\nabla}_{\mu} \sigma\right)-\frac{\Lambda}{k} \int d^{4} x \sqrt{-\bar{g}} e^{4 \sigma} .
$$


Then, the full effective action is given by

$$
S_{\text {eff }}=S_{\text {anom }}+S_{c l}
$$

with the additional criterion that terms independent of $\sigma$ are to be dropped out.

It has been argued in [2] — where the study of the dynamics of the conformal factor $\sigma$ on a flat fiducial background has been carried out - that this theory (which is ultraviolet renormalizable) describes, in its infrared stable fixed point, 4d quantum gravity at large distances (i.e., infrared quantum gravity). The exact anomalous scaling dimension of the conformal factor at the fixed point has been derived in [2] in the same way as is commonly done for $2 \mathrm{~d}$ induced gravity [9].

An indication of the necessity of an exact treatment of the conformal factor dynamics in the infrared region comes from the study of the graviton propagator in De Sitter space ${ }^{3}$. It has been shown $[10,11]$ that the graviton propagator is not bounded at large distances, where the main contribution is given by the conformal part [11].

Now, as in [6], one can generalize the theory with the action (6) to the case of a curved fiducial background, in order to construct a multiplicatively renormalizable theory in curved space-time:

$$
\begin{aligned}
S & =\int d^{4} x \sqrt{-g}\left\{-\frac{\theta^{2}}{(4 \pi)^{2}} \sigma \square^{2} \sigma+\sigma\left[\xi_{1} R^{\mu \nu} \nabla_{\mu} \nabla_{\nu}+\xi_{2} R \square+\xi_{3}\left(\nabla_{\mu} R\right) \nabla^{\mu}\right] \sigma\right. \\
& -\zeta\left[2 \alpha\left(\nabla_{\mu} \sigma\right)\left(\nabla^{\mu} \sigma\right) \square \sigma+\alpha^{2}\left(\left(\nabla_{\mu} \sigma\right)\left(\nabla^{\mu} \sigma\right)\right)^{2}\right]+\frac{\eta_{1}}{\alpha^{2}} e^{2 \alpha \sigma} R+\eta_{2} R\left(\nabla_{\mu} \sigma\right)\left(\nabla^{\mu} \sigma\right) \\
& \left.+\gamma e^{2 \alpha \sigma}\left(\nabla_{\mu} \sigma\right)\left(\nabla^{\mu} \sigma\right)-\frac{\lambda}{\alpha^{2}} e^{4 \alpha \sigma}+\frac{a_{1}}{\alpha^{2}} R_{\mu \nu}^{2}+\frac{a_{2}}{\alpha^{2}} G+\frac{a_{3}}{\alpha^{2}} R^{2}+\frac{a_{4}}{\alpha^{2}} \square R\right\} .
\end{aligned}
$$

A few remarks are in order. First, we have dropped the bar of the metric tensor, geometrical invariants and derivatives. Also, as in ref. [2], we have assumed that the $e^{\sigma}$ acquires a scaling dimension $\alpha$, and we have done the transformation $\sigma \rightarrow \sigma \alpha$. The presence of a term linear in $\sigma$ which cancels all terms linear in $\sigma$, both at the classical and at the quantum level, is also assumed [2] (such a trick is standard in quantum field theory and can be found in textbooks $[15])$.

In the theory with the action (7), the coupling constants $\xi_{1}, \xi_{2}, \xi_{3}, \zeta, \eta_{1}, \theta^{2}, \eta_{2}, \gamma, \lambda, a_{1}$, $a_{2}, a_{3}$ and $a_{4}$ are arbitrary. In order to obtain a correspondence with the $S_{\text {eff }}$ given by (6), one is bound to choose them in the following way

$$
\frac{\theta^{2}}{(4 \pi)^{2}}=2 b+3 b^{\prime \prime}, \quad \zeta=2 b+2 b^{\prime}+3 b^{\prime \prime}, \quad \gamma=\frac{3}{k}, \quad \lambda=\frac{\Lambda}{k},
$$

\footnotetext{
${ }^{3}$ Recently, an interesting approach, the so-called mean-field quantum gravity, has been discussed in De Sitter space [12].
} 


$$
\begin{aligned}
& a_{1}=a_{2}=a_{3}=a_{4}=0, \quad \eta_{1}=\frac{1}{2 k}, \quad \eta_{2}=b^{\prime \prime}+\frac{2}{3}\left(b+b^{\prime}\right), \\
& \xi_{1}=2\left(\zeta-\frac{\theta^{2}}{(4 \pi)^{2}}\right), \quad \xi_{2}=-\zeta+\frac{2}{3} \frac{\theta^{2}}{(4 \pi)^{2}}, \quad \xi_{3}=-\frac{1}{3} \frac{\theta^{2}}{(4 \pi)^{2}} .
\end{aligned}
$$

Notice that, given in the form (7) with arbitrary coupling constants, this theory is multiplicatively renormalizable in curved space-time (for a general discussion, see [1]). In the infrared stable fixed point $\zeta=0$ [2] (the only case we shall here discuss), and with the parameters (8), the theory describes qualitatively well the infrared sector of quantum gravity. Moreover, at $\zeta=0$ the theory given by (7) is still multiplicatively renormalizable in curved space-time. It is interesting to observe also, that the theory under consideration is probably connected with the self-dual limit of higher derivative quantum gravity $[13,14]$.

Of course, the model given by the action (7) contains higher-derivative terms. Hence, it is expected to be non-unitary at the quantum level. However, most probably the unitarity can be restored as in the case of $c>25$ non-critical strings [16]. And, from another point of view, only fundamental theories should be demanded to be unitary, and not effective theories in which the effects of spin-two gravitational modes are frozen.

We now explicitly give the $\beta$-functions for the theory (7), corresponding to the infrared stable fixed point $\zeta=0$. We shall write here only the beta functions for the coupling constants connected with the field $\sigma$ (taking into account the classical scaling dimension for $e^{\sigma}$ ). The $\beta$-functions for the $R^{2}$-terms (vacuum energy) are not so important, owing to the fact that the vacuum energy on a fixed background can actually be considered as a constant. We have

$$
\begin{aligned}
& \beta_{\gamma}=\left(2-2 \alpha+\frac{2 \alpha^{2}}{\theta^{2}}\right) \gamma, \\
& \beta_{\lambda}=\left(4-4 \alpha+\frac{8 \alpha^{2}}{\theta^{2}}\right) \lambda-\frac{8 \pi^{2} \alpha^{2} \gamma^{2}}{\theta^{4}}\left(1+\frac{4 \alpha^{2}}{\theta^{2}}+\frac{6 \alpha^{4}}{\theta^{4}}\right), \\
& \beta_{\eta_{1}}=\left(2-2 \alpha+\frac{2 \alpha^{2}}{\theta^{2}}\right) \eta_{1}+\frac{\alpha^{2} \gamma}{6 \theta^{2}}-\frac{(4 \pi)^{2} \alpha^{2} \gamma}{\theta^{4}}\left(\frac{\xi_{1}}{4}+\xi_{2}\right),
\end{aligned}
$$

where $\beta_{\gamma}$ and $\beta_{\lambda}$ have been calculated in [2] and are exact results, $\beta_{\eta_{1}}$ has been obtained in the one-loop approximation [6], and the remaining $\beta$-functions (i.e., those for $\xi_{1}, \xi_{2}, \xi_{3}$ and $\eta_{2}$ ) do not appear in the one-loop approximation [6]. As has been argued in ref. [2], the trace of the energy-momentum tensor for the $\sigma$-field sector must vanish, in direct analogy with $2 \mathrm{~d}$ gravity [9]. Hence, the $\beta$-functions (9) must necessarily vanish. From the conditions $\beta_{\gamma}=0$ and $\beta_{\lambda}=0$, one gets [2]

$$
\alpha_{ \pm}=\frac{1 \pm \sqrt{1-4 / \theta^{2}}}{2 / \theta^{2}}, \quad \frac{\lambda}{\gamma^{2}}=9 k \Lambda=\frac{2 \pi^{2}}{\theta^{2}}\left(1+\frac{4 \alpha^{2}}{\theta^{2}}+\frac{6 \alpha^{4}}{\theta^{4}}\right) .
$$




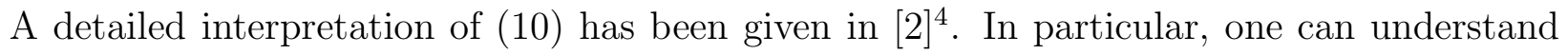
from this expression that the value $\theta_{c r}^{2}=4$ corresponds to the $c=1$ string, where a phase transition could be expected. Thus, it is meaningful to pose the question: is this indeed the case in the theory under discussion? Does this model actually exhibit a phase transition? In what follows we shall address this point.

As first step, we restrict ourselves to flat space and calculate the Coleman-Weinberg potential for the theory (7). Let us denote $e^{\alpha \sigma}=\phi$. Then, the one-loop effective potential for the theory (7) is given by

$$
V^{(1)}(\phi)=\frac{\lambda}{\alpha^{2}} \phi^{4}+B \phi^{4}+A \phi^{2}+\frac{1}{2} \int \frac{d^{4} k}{(2 \pi)^{4}}\left[\ln \left(1+\frac{A_{1} \phi^{2}}{k^{2}}\right)+\ln \left(1+\frac{A_{2} \phi^{2}}{k^{2}}\right)\right],
$$

where

$$
A_{1,2}=\frac{(4 \pi)^{2}}{2 \theta^{2}}\left(\gamma \pm \sqrt{\gamma^{2}-32 \frac{\lambda \theta^{2}}{(4 \pi)^{2}}}\right)
$$

and $A$ and $B$ are renormalization constants. With the same renormalization conditions as in the paper by Coleman and Weinberg [17], one can immediately find the values of $A$ and $B$. Finally, we obtain the following effective potential:

$$
V^{(1)}\left(e^{\alpha \sigma}\right)=\frac{\lambda}{\alpha^{2}} e^{4 \alpha \sigma}+\frac{1}{2}\left[\frac{\gamma^{2}(4 \pi)^{2}}{2 \theta^{4}}-\frac{8 \lambda}{\theta^{2}}\right] e^{4 \alpha \sigma}\left(\frac{\sigma}{\sigma_{0}}-\frac{25}{6}\right),
$$

where $\sigma_{0}$ is the normalization point. In particular, from (12) one can easily obtain the value of $\sigma$ corresponding to Coleman-Weinberg's spontaneous symmetry breaking

$$
\frac{\sigma}{\sigma_{0}}=-\frac{\lambda}{\alpha^{2}}\left\{\frac{1}{2}\left[\frac{\gamma^{2}(4 \pi)^{2}}{2 \theta^{4}}-\frac{8 \lambda}{\theta^{2}}\right]\right\}^{-1}+\frac{25}{6}-\frac{1}{4 \alpha}
$$

or, if we take into account (10),

$$
\frac{\sigma}{\sigma_{0}}=-\frac{\lambda}{2(1-\alpha)}+\frac{25}{6}-\frac{1}{4 \alpha}
$$

where $\lambda$ is given by the second expression (10). Hence, we can estimate the value of $\sigma$ corresponding to spontaneous symmetry breaking.

Consider now the one-loop effective potential for the theory (7) on a curved background. We shall only consider the linear curvature approximation. There are different ways of doing this calculation. One of them is to use the renormalization group method and find the one-loop effective potential. Alternatively, we can perform the calculations on a De Sitter background, where $\zeta$-function regularization works very well, and expand the final answer

\footnotetext{
${ }^{4}$ Notice that, taking into account (10) and the values of $\xi_{1}$ and $\xi_{2}$ from (8), equation $\beta_{\eta_{1}}=0$ is fulfilled automatically
} 
taking into account all terms up to the ones linear in the curvature. Both procedures give the same answer (provided the same renormalization conditions are used). Since the details of such manipulations are quite standard (for instance, they can be found in [1]), we shall here only give the final result

$$
\begin{aligned}
V^{(1)}(\phi) & =\frac{\lambda}{\alpha^{2}} \phi^{4}+\frac{1}{2}\left[\frac{\gamma^{2}(4 \pi)^{2}}{2 \theta^{4}}-\frac{8 \lambda}{\theta^{2}}\right] \phi^{4}\left(\ln \frac{\phi^{2}}{\mu^{2}}-\frac{25}{6}\right)-\frac{\eta_{1}}{\alpha^{2}} \phi^{2} R \\
& +\frac{1}{2}\left[\frac{1}{\theta^{2}}\left(2 \eta_{1}+\frac{\gamma}{6}\right)-\frac{(4 \pi)^{2} \gamma}{\theta^{4}}\left(\frac{\xi_{1}}{4}+\xi_{2}\right)\right] \phi^{2} R\left(\ln \frac{\phi^{2}}{\mu^{2}}-3\right)
\end{aligned}
$$

where $\phi^{2} \equiv e^{2 \alpha \sigma}, \mu^{2} \equiv e^{2 \alpha \sigma_{0}}$ and we assume that $\phi^{2}>>|R|$ (the linear curvature approximation). As one can see immediately, the first two terms represent the Coleman-Weinberg potential calculated above, while the remaining terms provide the curvature correction.

We now concentrate on the possibility of a curvature-induced phase transition (see [1] for a description of the general situation). An interesting possibility is that of a first-order phase transition, when the order parameter $\langle\phi\rangle$ experiences a quick change for some critial value of the curvature, $R_{c}$. The standard condition of such a phase transition are:

$$
V^{(1)}\left(\phi_{c}, R_{c}\right)=0,\left.\quad \frac{\partial V^{(1)}}{\partial \phi_{c}}\right|_{\phi_{c}, R_{c}}=0,\left.\quad \frac{\partial^{2} V^{(1)}}{\partial \phi_{c}^{2}}\right|_{\phi_{c}, R_{c}}>0 .
$$

Let us discuss a few different cases. Impose the following (rather natural) restrictions on the parameters in (15): $\gamma^{2}(4 \pi)^{2}>>|\lambda|, \eta_{1} \simeq \gamma, \alpha^{2}=\theta^{2}=1, \gamma<<1,4 \pi^{2}\left|\xi_{1} / 4+\xi_{2}\right|>1$ and $4 \pi^{2}\left|\gamma\left(\xi_{1} / 4+\xi_{2}\right)\right|<<1$. Then it is immediate that a phase transition is possible, and that

$$
e^{2 \alpha\left(\sigma_{c}-\sigma_{0}\right)} \sim e^{3}, \quad\left|R_{c}\right| e^{-2 \alpha \sigma_{0}} \sim-\frac{4 \pi^{2} \gamma^{2}}{\eta_{1} \operatorname{sgn}\left(R_{c}\right)} e^{3} .
$$

We can also consider the set of parameters (15) which correspond to infrared quantum gravity:

$$
\frac{\gamma}{6 \theta^{2}}-\frac{(4 \pi)^{2} \gamma}{\theta^{4}}\left(\frac{\xi_{1}}{4}+\xi_{2}\right)=0, \quad \eta_{1}=\frac{\gamma}{6}
$$

In particular, with the following choice: $\alpha=1, \theta^{2}>>1, \gamma<<1, \gamma^{2} / \theta^{2} \sim \lambda<<1$, $\lambda>\gamma^{2} \pi^{2} / \theta^{2}$, we get

$$
e^{2 \alpha\left(\sigma_{c}-\sigma_{0}\right)} \sim e^{-\theta^{2} / 4}, \quad\left|R_{c}\right| e^{-2 \alpha \sigma_{0}} \sim-\frac{32 \lambda}{\theta^{2} \gamma \operatorname{sgn}\left(R_{c}\right)} e^{-\theta^{2} / 4}
$$

The phase transition is clearly seen for standard values of the parameters which satisfy these conditions (Fig. 1). It takes place for a very reasonable value of the curvature $\left(R_{c} \simeq 1\right)$. To be noticed is the fact that, also for the critical case, $\theta_{c r}^{2}=4, \alpha_{c r}^{2}=2$, we do find a phase transition in our approach, but this happens only for sufficiently high values of the curvature ( $R_{c} \simeq 100$, see Fig. 2 ). However, our approximation is presumably not good enough for very 
large curvature, and higher order corrections on the curvature should be taken into account in that case. Also interesting is the following situation, corresponding to the conditions in our present universe: $\theta^{2}=1$ and $\alpha^{2}=1$. The phase transition occurs here for even higher values of the curvature $\left(R_{c} \simeq 500\right)$. It is represented in Fig. 3 .

The main qualitative result in this paper is the explicit proof that for some values of the parameters the conformal sector of quantum gravity can yield a curvature induced phase transition. Also important is the observation that in the case of an external background with torsion, the conformal sector of quantum gravity with torsion [18] can lead to a torsioninduced phase transition.

A final remark about possible phase transitions at non-zero temperature. It is known that the trace anomaly does not change at non-zero temperature. Therefore, if we consider the flat fiducial background with a compactified time dimension, the effective potential is given again by (11), where $d k_{0}$ is to be changed in the standard way. If we add to the classical potential a mass-like term of the kind $-\left[\mathrm{m}^{2} /\left(2 \alpha^{2}\right)\right] e^{2 \alpha \sigma}$, then $A_{1}$ and $A_{2}$ are slightly modified and we can find the critical inverse temperature as in ref. [19]:

$$
\beta_{c}^{2}=-\frac{\gamma(4 \pi)^{2} \alpha^{2}}{12 \theta^{2} m^{2}}
$$

where $m^{2}$ is an unphysical, negative mass [19]. Therefore, by adding a mass-like term to the effective potential we get the possibility of a phase transition at non-zero temperature.

In summary, we have shown that the trace-anomaly-induced dynamics (infrared quantum gravity) has a very rich phase structure and can undergo a phase transition induced by the curvature. It would be interesting to understand if such a phase transition has really taken place in the early universe and, if so, if it can lead to some important cosmological consequences.

\section{Acknowledgments}

S.D.O. wishes to thank I. Antoniadis for extremely valuable discussions and the Particle Group at Hiroshima University for kind hospitality. S.D.O. has been supported by JSPS (Japan) and E.E. by DGICYT (Spain), research project PB90-0022. 


\section{References}

[1] I.L. Buchbinder, S.D. Odintsov and I.L. Shapiro, Effective Action in Quantum Gravity, IOP Publishing, Bristol and Philadelphia, 1992.

[2] I. Antoniadis and E. Mottola, Phys. Rev. D45 (1992) 2013.

[3] S. Weinberg, Rev. Mod. Phys. 61 (1989) 1.

[4] A.M. Polyakov, Phys. Lett. B103 (1981) 207.

[5] S.D. Odintsov and I.L. Shapiro, Class. Quant. Grav. 8 (1991) L57.

[6] S.D. Odintsov, Z. Phys. C54 (1992) 531.

[7] I. Antoniadis, P.O. Mazur and E. Mottola, preprint LA-UR-92-1483, 1992.

[8] M.J. Duff, Nucl. Phys. B125 (1977) 334; S. Deser, M.J. Duff and C.J. Isham, Nucl. Phys. B111 (1976) 45.

[9] V.G. Knizhnik, A.M. Polyakov and A.B. Zamolodchikov, Mod. Phys. Lett. A3 (1988) 819; J. Distler and H. Kawai, Nucl. Phys. B321 (1989) 509; F. David, Mod. Phys. Lett. A3 (1988) 1651.

[10] I. Antoniadis, J. Iliopoulos and T.N. Tomaras, Phys. Rev. Lett. 56 (1986) 1319; E.G. Floratos, J. Iliopoulos and T.N. Tomaras, Phys. Lett. B197 (1987) 373; T.R. Taylor and G. Veneziano, Phys. Lett. B212 (1988) 147.

[11] I. Antoniadis and E. Mottola, J. Math. Phys. 32 (1991) 1037.

[12] R. Floreanini and R. Percacci, Phys. Rev. D46 (1992) 1566.

[13] C. Schmidhuber, preprint CALT-68-1745, 1992.

[14] E. Elizalde and S.D. Odintsov, preprint hep-th 9205048, to appear in Int. J. Mod. Phys. D.

[15] T. Muta, Foundations of Quantum Chromodynamics, World Scientific, Singapore, 1987.

[16] I. Antoniadis, C. Bachas, J. Ellis and D.V. Nanopoulos, Nucl. Phys. B328 (1989) 117; R.C. Myers, Phys. Lett. B199 (1987) 371.

[17] S. Coleman and E. Weinberg, Phys. Rev. D7 (1973) 1888.

[18] I. Antoniadis and S.D. Odintsov, paper in preparation.

[19] L. Dolan and R. Jackiw, Phys. Rev. D9 (1974) 2491. 


\section{Figure captions.}

Fig. 1. The effective potential at one-loop, $V^{(1)}(\phi)$ (eq. (15)), represented as a function of $\phi$ for several values of the curvature $R$. We have taken a typical set of parameters corresponding to infrared quantum gravity (eq. (18)), in particular: $\alpha=1, \theta^{2}=10^{2}$, $\gamma=10^{-2}$ and $\lambda=10^{-3}$. The phase transition appears already for quite small values of $R$ (in this example $R_{c} \simeq 1$ ).

Fig. 2. The same as in Fig. 1, but for the values of constants corresponding now to the critical case: $\theta_{c r}^{2}=4, \alpha_{c r}^{2}=2$, and $\lambda / \gamma^{2}$ as given through eq. (10). The phase transition appears only for very high values of the curvature $\left(R_{c} \simeq 100\right)$.

Fig. 3. The same as in Fig. 1, but for the following values of the parameters (which correspond to our present universe): $\theta^{2}=1, \alpha^{2}=1$ and $\gamma=3 / k$, where $1 / 2 k=1 / 16 \pi G$, being $G$ the gravitational constant. The phase transition appears at even higher values of the curvature $\left(R_{c} \simeq 500\right)$. 